\title{
Torsten Groth, Gerhard P. Krejci \& Stefan Günther (Hrsg.) New Organizing. Wie Großorganisationen Agilität, Holacracy \& Co. einführen - und was man daraus lernen kann.
}

\author{
Heidelberg: Carl Auer Systeme 2021 ISBN: 978-3-8479-0402-5
}

\author{
Ewald E. Krainz'
}

Angenommen: 10. Januar 2022 / Online publiziert: 20. Januar 2022

(c) Der/die Autor(en) 2022

\section{Torsten Groth, Gerhard P. Krejci \& Stefan Günther (eds.) New Organizing. How large organizations implement agility, holacracy \& Co. - and what we can learn from it}

Heidelberg: Carl Auer Systeme 2021 ISBN: 978-3-8479-0402-5

Der Untertitel lässt eine leicht ironische Distanz zum Untersuchungsgegenstand erkennen, was an sich schon sympathisch ist. Jedenfalls hat man es beim vorliegenden Buch nicht mit der Sorte Literatur zu tun, wo Ansätze angepriesen werden. Andererseits läuft das Vorgebrachte aber auch nicht auf eine (nicht schwer zu habende) kritische Gesamtverwerfung der diversen Ansätze hinaus, die von den Herausgebern unter dem Sammelbegriff New Organizing zusammengefasst werden. Vielmehr ging es den Herausgebern darum, Organisationen genauer in Augenschein zu nehmen, deren jüngere Entwicklung von Bemühungen gekennzeichnet war bzw. ist, sich unter Rückgriff auf entsprechende Konzepte (neu) auszurichten. In diesem Sinn ist das Buch eine theoretisch umrahmte empirische Studie über aktuelle Organisationszustände und Veränderungsprozesse.

New Organizing ist das Ergebnis eines breit angelegten Forschungsprojekts, an dem sich eine Community von Interessierten beteiligte, entsprechend energetisiert von der Simon Weber Friends (SWF) „Denkwerkstatt“, im Besonderen von den drei Herausgebern. In Summe waren über 40 Personen beteiligt (zu viele, um sie hier alle namentlich zu nennen), vielfach SWF-Weiterbildungs-Alumni, die im Wesentlichen mit unterschiedlichen Akzentuierungen der Consultingbranche zuzurechnen sind. In 13 kompakten

\footnotetext{
$\triangle$ Dr. Ewald E. Krainz

office@ewaldkrainz.at

1 University of Klagenfurt, Klagenfurt, Österreich
}

Falldarstellungen (verfasst zu zweit bis zu fünft) wird auf der Basis von Interviews mit Beteiligten dargestellt, was sich in den untersuchten Organisationen abgespielt hat, die sich entsprechenden Übungen unterzogen haben.

Die Falldarstellungen sind jede für sich genommen schon interessant. Keineswegs handelt es sich um bloße Erfolgsgeschichten. Vielmehr wird deutlich in welchem Spannungsfeld zwischen Konzept-Verheißungen, Umsetzungen und tatsächlich Erreichtem man sich in den Organisationen befindet. Wenn Verantwortliche Organisationskonzepte implementieren wollen, ist es ja (hoffentlich) nicht so, dass sie sich gedankenlos modischen Trends ausliefern, und es ist ihnen auch zuzutrauen, einigermaßen ,,vernünftig“ $\mathrm{zu}$ manövrieren. Was aber ist mit den unbeabsichtigten Folgen, den unerwünschten Nebenwirkungen und jenen emergenten Phänomenen, an die man nicht gedacht hat bzw. auch gar nicht denken konnte? Wie steht es diesbezüglich mit der Antwortfähigkeit? Das sind Fragen, die sich gewollt oder nicht aufwerfen und die nicht selten offen bleiben.

Die herausgeberische Komposition, die der Idee zu diesem Buch zu Grunde liegt, ist von seltener Güte. Herausgeber-Bände sind in der Regel Ansammlungen von Einzelbeiträgen, mehr oder weniger ausführlich von einem Editorial ein-begleitet.

Anders hier. Zunächst wird Einblick in den Zugang der Herausgeber gegeben, die sich auch in „,choreografischen“ Überlegungen niedergeschlagen haben, unter welchen Gesichtspunkten die einzelnen Organisationen als Fälle zu be- 
obachten sind. Ich führe sie an und kommentiere assoziativparaphrasierend:

- Mit welcher Leitidee wurden neue Organisationsformen eingeführt? - Die Versionen, welche die Motivationslage beschreiben, die tatsächliche wie auch die offiziell verkündete, sind nicht immer deckungsgleich.

- Mit welcher Bezeichnung wurde der neue Ansatz versehen? - Der verwendeten Sprache gebührt Aufmerksamkeit, denn die Semantik der Namen transportiert bisweilen unterschwellige Neben-Bedeutungen, nicht selten unfreiwillig komisch. (Ein Glossar der gängigen Begriffe findet sich am Ende des Buchs.)

- Welche Auswirkungen zeitigten die Aktivitäten? - In vielen Anwendungslagen ist es schlechte Praxis im Management, immer etwas „Neues“ zu inszenieren, bevor man sich noch hinlänglich darüber vergewissern konnte, was die vorangegangene Initiative erbracht hat; so, als wäre es ein „Lebenszeichen“ (incl. Existenzrechtfertigung) des Managements, die Organisation auf Trab zu halten.

- Welche Unterstützungen - seitens HR u/o externer Beratung haben mitgeholfen, den neuen Ansatz ins Leben zu bringen? - Das ist eine kritische Frage für die Consultingbranche, aber auch für das HR-Personal; es ist nicht zwangsläufig davon auszugehen, dass hinsichtlich der Situationsauffassung die Übereinstimmung mit den jeweiligen Oberleitungen in den Organisationen automatisch gegeben ist.

- Wie verlief der Implementierungsprozess? - „Reibungslos" oder nicht, und wenn nicht, was ist die Natur der „Reibungen“ und was sagt das über die Organisation und ihre Mikropolitiken?

- Gab es eine prozessbegleitende Reflexion? - Es könnte ja auch sein, dass sich die Initiative darin erschöpft, sie in Gang gebracht zu haben.

- Und schließlich die Frage aller Fragen: Was hat man gelernt? - Und welche Konsequenzen wurden daraus gezogen?

Diese vorformulierten Blickwinkel waren für die empirischen Beobachtungen orientierend, ohne dass damit eine Liste von Fragen taxativ abzuarbeiten war. Trotz der Vorformulierung (weniger im Sinne einer Vorgabe als einer Anregung) hat man beim Lesen das Gefühl, dass den Eigenheiten der jeweils untersuchten Organisationen in den Darstellungen Rechnung getragen werden konnte.

Des Weiteren haben sich die Herausgeber darüber Gedanken gemacht, wie das zusammengetragene Fallmaterial strukturiert und ausgewertet werden kann. Was die Strukturierung betrifft, haben sie einen „Fahrplan“ hergestellt, der zwei Aspekte bedenkt: Wie existenziell notwendig war die Veränderung? Und war sie eher top-down oder bottom-up initiiert? Damit ergab sich ein Raster, das die Anordnung der einzelnen Beiträge nacheinander zur Folge hatte. Nach jedem einzelnen Beitrag folgt immer ein Kurzkommentar der Herausgeber mit überleitenden Worten zur nächsten Fallgeschichte. Das ist zweifellos eine anerkennenswerte „ästhetische“ Bemühung in Richtung eines Gesamtnarrativs, man kann in die einzelnen Beiträge aber auch kreuz und quer einsteigen, ganz nach Belieben oder Zufall.

Die letzten zwei Beiträge heben sich von den Fallstudien ab. Der eine kommentiert den Umstand, dass es ja auch Unternehmen gibt, die sozusagen „konventionell“, also ohne das Spiel New Organizing mitzuspielen - fast müsste man sagen: dennoch - anhaltend erfolgreich sind. Dass es solche gibt, sollte man nicht vergessen. Der andere fokussiert auf die Forschungscommunity selbst und welche Spiegelungsphänomene aufgetreten sind (hinsichtlich Führungs-, Team- und Organisationsfragen). Das hinterlässt den Eindruck, dass hier auch dem Anspruch an Selbstreflexion (deren Fehlen in Fallbeschreibungen als Mangel benannt wird) nachgekommen wurde, sicherlich zum Nutzen des Forschungsprojekts.

Von systemischer Organisationstheorie inspiriert nennen die Herausgeber ihre Herangehensweise. Die Zurücknahme des Theorieanspruchs von „systemisch“ zu „systemisch inspiriert" hat etwas Wohltuendes. So wird nicht - wie in vielen Beispielen systemischer Literatur - innerhalb der systemischen Denkschemata argumentiert (so als müsste man am eigenen Beispiel demonstrieren, wie „Autopoiesis“ in der Produktion theoretischer Literatur geht), sodass einem der dadurch entstehende Langeweile-Faktor erspart bleibt. Vielmehr werden - trotz deutlicher Beheimatung im Systemischen - auch Bezüge zu anderen relevanten Quellen hergestellt, so etwa zu Weicks Begriff des Organizing oder Mintzbergs Begriff des Managing. Aber es geht beim Forschungsprojekt ohnehin nicht um die Erprobung der Systemtheorie für das Untersuchungsfeld, im Vordergrund der Aufmerksamkeit stehen die empirischen Gegebenheiten.

Hinsichtlich der methodischen Vorgangsweise haben die Herausgeber beabsichtigt oder unbeabsichtigt sich selbst zu Beobachtern zweiter Ordnung gemacht. Das kulminiert in einer schönen Zusammenfassung, welche Problemlagen beim New Organizing immer auftreten, wahrscheinlich auch beim konventionellen Organisieren (die Wörter kann man sich merken): Organisationstrivialisierung, Führungsvergessenheit, Teamentkoppelung und Beratungsvergessenheit. Für den Umgang damit, sei es aus Managementoder Beratungsperspektive, empfehlen die Autoren im Wesentlichen eine widerspruchsoffene (latent auch machtkritische) Haltung. Postmodisch soll man agieren, meinen die Herausgeber, Ansätze sind allenfalls Anregungen, maßzuschneidern für die jeweils gegeneben Situationen, keine normativen Konzepte.

Insgesamt ist den Herausgebern und allen Mitwirkenden mit dem Buch ein in Anspruch, Durchführung und Ergebnis 
originelles Werk gelungen, dem viele Leserinnen und Leser zu wünschen sind.

Funding Open access funding provided by University of Klagenfurt.

Open Access Dieser Artikel wird unter der Creative Commons Namensnennung 4.0 International Lizenz veröffentlicht, welche die Nutzung, Vervielfältigung, Bearbeitung, Verbreitung und Wiedergabe in jeglichem Medium und Format erlaubt, sofern Sie den/die ursprünglichen Autor(en) und die Quelle ordnungsgemäß nennen, einen Link zur Creative Commons Lizenz beifügen und angeben, ob Änderungen vorgenommen wurden.

Die in diesem Artikel enthaltenen Bilder und sonstiges Drittmaterial unterliegen ebenfalls der genannten Creative Commons Lizenz, sofern sich aus der Abbildungslegende nichts anderes ergibt. Sofern das betreffende Material nicht unter der genannten Creative Commons Lizenz steht und die betreffende Handlung nicht nach gesetzlichen Vorschriften erlaubt ist, ist für die oben aufgeführten Weiterverwendungen des Materials die Einwilligung des jeweiligen Rechteinhabers einzuholen.
Weitere Details zur Lizenz entnehmen Sie bitte der Lizenzinformation auf http://creativecommons.org/licenses/by/4.0/deed.de.

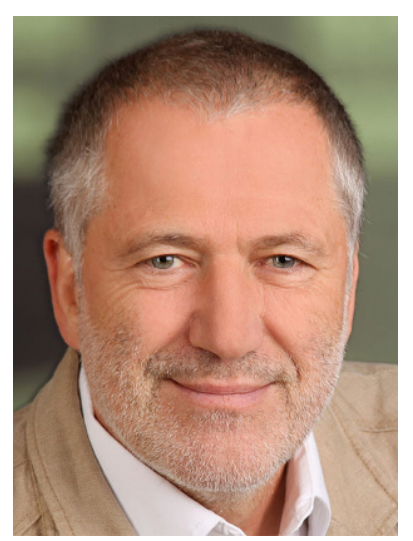

Dr. Ewald E. Krainz Ao. Univ.Prof. i.R. für Gruppendynamik und Organisationsentwicklung Univ. Klagenfurt; Lehrtrainer und Lehrberater ÖGGO (Österreichische Gesellschaft für Gruppendynamik und Organisationsberatung); zu Philosophie und Arbeitsschwerpunkten siehe www.ewaldkrainz.at. 